\title{
HEAT FLUX FORMULATION FOR 1D DUAL-PHASE LAG EQUATION
}

\author{
Ewa Majchrzak, Grażyna Kałuża \\ Institute of Computational Mechanics and Engineering \\ Silesian University of Technology \\ Gliwice, Poland \\ ewa.majchrzak@polsl.pl,grazyna.kaluza@polsl.pl
}

\begin{abstract}
The thin metal film subjected to the ultra-short laser pulse is analyzed. Heat transfer processes occurring in the domain considered are described by the dual-phase lag model in which the unknown is the heat flux, not, as usual, temperature. This approach is especially convenient in the case of Neumann boundary conditions, which are taken into account here. The mathematical model supplemented by initial conditions is solved using the explicit scheme of finite difference method. In the final part of the paper the examples of computations are shown and the conclusions are formulated.
\end{abstract}

Keywords: dual-phase lag equation, finite difference method, thin metal film

\section{Introduction}

The $1 \mathrm{D}$ Fourier equation is given as

$$
c \frac{\partial T(x, t)}{\partial t}=-\frac{\partial q(x, t)}{\partial x}+Q(x, t)
$$

where $c$ is the volumetric specific heat of tissue, $q(x, t)$ is the heat flux, $Q(x, t)$ is the capacity of internal heat sources, $T$ is the temperature, $x$ is the spatial co-ordinate and $t$ is the time.

Heat transfer at the macroscale is described by the equation based on the classical Fourier law

$$
q(x, t)=-\lambda \frac{\partial T(x, t)}{\partial x}
$$

where $\lambda$ is the thermal conductivity of material. Introducing (2) into (1), under the assumption that $\lambda$ is a constant, one obtains the Fourier equation in the form

$$
c \frac{\partial T(x, t)}{\partial t}=\lambda \nabla^{2} T(x, t)+Q(x, t)
$$


Heat transfer at the microscale can be described, among others, by the dual-phase-lag model [1-3] based on the assumption that

$$
q\left(x, t+\tau_{q}\right)=-\lambda \frac{\partial T\left(x, t+\tau_{T}\right)}{\partial x}
$$

where $\tau_{q}$ is the relaxation time, $\tau_{T}$ is the thermalization time.

Taking into account the first order Taylor expansions for $q$ and $T$

$$
q(x, t)+\tau_{q} \frac{\partial q(x, t)}{\partial t}=-\lambda \frac{\partial T(x, t)}{\partial x}-\lambda \tau_{T} \frac{\partial^{2} T(x, t)}{\partial t \partial x}
$$

one obtains the following form of the dual-phase lag equation

$$
c\left[\frac{\partial T(x, t)}{\partial t}+\tau_{q} \frac{\partial^{2} T(x, t)}{\partial t^{2}}\right]=\lambda \frac{\partial^{2} T(x, t)}{\partial x^{2}}+\lambda \tau_{T} \frac{\partial^{3} T(x, t)}{\partial t \partial x^{2}}+Q(x, t)+\tau_{q} \frac{\partial Q(x, t)}{\partial t}
$$

It should be pointed out that for $\tau_{q}=\tau_{T}=0$ the dual-phase-lag (DPL) equation (6) reduces to the Fourier equation (3).

Equation (6), in which the temperature is unknown, is widely used in the modeling of heat transfer in the micro scale, e.g. [1, 3-5]. This paper presents the dual-phase lag equation in which the heat flux is unknown [6]. It is very convenient, especially in the case of the Neumann boundary conditions.

\section{Dual-phase lag equation with an unknown heat flux}

Equation (5) is differentiated with respect to time

$$
\frac{\partial q(x, t)}{\partial t}+\tau_{q} \frac{\partial^{2} q(x, t)}{\partial t^{2}}=-\lambda \frac{\partial^{2} T(x, t)}{\partial t \partial x}-\lambda \tau_{T} \frac{\partial^{3} T(x, t)}{\partial t^{2} \partial x}
$$

or

$$
\frac{\partial q(x, t)}{\partial t}+\tau_{q} \frac{\partial^{2} q(x, t)}{\partial t^{2}}=-\lambda \frac{\partial}{\partial x}\left[\frac{\partial T(x, t)}{\partial t}\right]-\lambda \tau_{T} \frac{\partial^{2}}{\partial x \partial t}\left[\frac{\partial T(x, t)}{\partial t}\right]
$$

From equation (1) results that

$$
\frac{\partial T(x, t)}{\partial t}=-\frac{1}{c} \frac{\partial q(x, t)}{\partial x}+\frac{1}{c} Q(x, t)
$$


Introducing (9) into (8) one obtains

$$
\begin{aligned}
& \frac{\partial q(x, t)}{\partial t}+\tau_{q} \frac{\partial^{2} q(x, t)}{\partial t^{2}}=a \frac{\partial^{2} q(x, t)}{\partial x^{2}}+a \tau_{T} \frac{\partial^{3} q(x, t)}{\partial t \partial x^{2}}- \\
& a \frac{\partial Q(x, t)}{\partial x}-a \tau_{T} \frac{\partial^{2} Q(x, t)}{\partial t \partial x}
\end{aligned}
$$

where $a=\lambda c$ is the diffusion coefficient.

In summary, in the present approach, equation (10) supplemented by appropriate boundary initial conditions must be solved, and then the temperature is determined from equation (9).

\section{Formulation of the problem}

The thin metal film of thickness $L$ subjected to the ultra-short laser pulse is considered. Heat flux distribution is described by equation (10) in which the source function is defined as follows $[1,7]$

$$
Q(x, t)=\sqrt{\frac{\beta}{\pi}} \frac{1-R}{t_{p} \delta} I_{0} \exp \left[-\frac{x}{\delta}-\beta \frac{\left(t-2 t_{p}\right)^{2}}{t_{p}^{2}}\right]
$$

where $I_{0}$ is the laser intensity, $t_{p}$ is the characteristic time of a laser pulse, $\delta$ is the absorption depth, $R$ is the reflectivity of the irradiated surface and $\beta=4 \ln 2$. The equation (10) is supplemented by boundary conditions

$$
x=0: q(0, t)=0, \quad x=L: q(L, t)=0
$$

and initial ones

$$
t=0: \quad T(x, 0)=T_{p},\left.\quad \frac{\partial T(x, t)}{\partial t}\right|_{t=0}=0
$$

where $T_{p}$ is the initial temperature of thin film.

\section{Method of solution}

To solve the problem formulated the explicit scheme of the finite difference method $[4,5]$ is used. At first, the time discretization is introduced

$$
0=t^{0}<t^{1}<\ldots<t^{f-2}<t^{f-1}<t^{f}<\ldots<t^{F}<\infty
$$


Let $q^{f}=q(x, f \Delta t)$, where $\Delta t$ is the time step. Then, for time $t^{f}=f \Delta t(f \geq 2)$ the following approximate form of equation (10) can be taken into account

$$
\begin{aligned}
& \frac{q^{f}-q^{f-1}}{\Delta t}+\tau_{q} \frac{q^{f}-2 q^{f-1}+q^{f-2}}{(\Delta t)^{2}}=a \frac{\partial^{2} q^{f-1}}{\partial x^{2}}+\frac{a \tau_{T}}{\Delta t}\left(\frac{\partial^{2} q^{f-1}}{\partial x^{2}}-\frac{\partial^{2} q^{f-2}}{\partial x^{2}}\right)- \\
& a \frac{\partial Q^{f-1}}{\partial x}-a \tau_{T} \frac{\partial Z^{f-1}}{\partial x}
\end{aligned}
$$

or

$$
\begin{aligned}
& \frac{q^{f}-q^{f-1}}{\Delta t}+\tau_{q} \frac{q^{f}-2 q^{f-1}+q^{f-2}}{(\Delta t)^{2}}=\frac{a\left(\Delta t+\tau_{T}\right)}{\Delta t} \frac{\partial^{2} q^{f-1}}{\partial x^{2}}-\frac{a \tau_{T}}{\Delta t} \frac{\partial^{2} q^{f-2}}{\partial x^{2}}- \\
& a \frac{\partial Q^{f-1}}{\partial x}-a \tau_{T} \frac{\partial Z^{f-1}}{\partial x}
\end{aligned}
$$

where (cf. equation (11))

$$
Z(x, t)=\frac{\partial Q(x, t)}{\partial t}=-\sqrt{\frac{\beta}{\pi}} \frac{1-R}{\delta} \frac{2 \beta\left(t-2 t_{p}\right)}{t_{p}^{3}} I_{0} \exp \left[-\frac{x}{\delta}-\beta \frac{\left(t-2 t_{p}\right)^{2}}{t_{p}^{2}}\right]
$$

while $Z^{f-1}=Z(x,(f-1) \Delta t)$.

Next, the geometrical mesh with constant step $h$ is introduced and for the internal node $i$ one obtains

$$
\begin{aligned}
& \frac{q_{i}^{f}-q_{i}^{f-1}}{\Delta t}+\tau_{q} \frac{q_{i}^{f}-2 q_{i}^{f-1}+q_{i}^{f-2}}{(\Delta t)^{2}}=\frac{a\left(\Delta t+\tau_{T}\right)}{\Delta t} \frac{q_{i-1}^{f-1}-2 q_{i}^{f-1}+q_{i+1}^{f-1}}{h^{2}}- \\
& \frac{a \tau_{T}}{\Delta t} \frac{q_{i-1}^{f-2}-2 q_{i}^{f-2}+q_{i+1}^{f-2}}{h^{2}}-a \frac{Q_{i+1}^{f-1}-Q_{i-1}^{f-1}}{2 h}-a \tau_{T} \frac{Z_{i+1}^{f-1}-Z_{i-1}^{f-1}}{2 h}
\end{aligned}
$$

Equation (17) can be written in the form

$$
\begin{aligned}
& \frac{\Delta t+\tau_{q}}{(\Delta t)^{2}} q_{i}^{f}-\frac{\Delta t+2 \tau_{q}}{(\Delta t)^{2}} q_{i}^{f-1}+\frac{\tau_{q}}{(\Delta t)^{2}} q_{i}^{f-2}=-\frac{2 a\left(\Delta t+\tau_{T}\right)}{h^{2} \Delta t} q_{i}^{f-1}+ \\
& \frac{a\left(\Delta t+\tau_{T}\right)}{h^{2} \Delta t}\left(q_{i-1}^{f-1}+q_{i+1}^{f-1}\right)+\frac{2 a \tau_{T}}{h^{2} \Delta t} q_{i}^{f-2}-\frac{a \tau_{T}}{h^{2} \Delta t}\left(q_{i-1}^{f-2}+q_{i+1}^{f-2}\right) \\
& -\frac{a}{2 h}\left(Q_{i+1}^{f-1}-Q_{i-1}^{f-1}\right)-\frac{a \tau_{T}}{2 h}\left(Z_{i+1}^{f-1}-Z_{i-1}^{f-1}\right)
\end{aligned}
$$


or

$$
\begin{aligned}
& \frac{\Delta t+\tau_{q}}{(\Delta t)^{2}} q_{i}^{f}=\frac{h^{2}\left(\Delta t+2 \tau_{q}\right)-2 a \Delta t\left(\Delta t+\tau_{T}\right)}{h^{2}(\Delta t)^{2}} q_{i}^{f-1}+ \\
& \frac{a\left(\Delta t+\tau_{T}\right)}{h^{2} \Delta t}\left(q_{i-1}^{f-1}+q_{i+1}^{f-1}\right)+\frac{2 a \tau_{T} \Delta t-h^{2} \tau_{q}}{h^{2}(\Delta t)^{2}} q_{i}^{f-2}- \\
& \frac{a \tau_{T}}{h^{2} \Delta t}\left(q_{i-1}^{f-2}+q_{i+1}^{f-2}\right)-\frac{a}{2 h}\left(Q_{i+1}^{f-1}-Q_{i-1}^{f-1}\right)-\frac{a \tau_{T}}{2 h}\left(Z_{i+1}^{f-1}-Z_{i-1}^{f-1}\right)
\end{aligned}
$$

Finally, one obtains

$$
\begin{aligned}
& q_{i}^{f}=\frac{h^{2}\left(\Delta t+2 \tau_{q}\right)-2 a \Delta t\left(\Delta t+\tau_{T}\right)}{h^{2}\left(\Delta t+\tau_{q}\right)} q_{i}^{f-1}+\frac{a \Delta t\left(\Delta t+\tau_{T}\right)}{h^{2}\left(\Delta t+\tau_{q}\right)}\left(q_{i-1}^{f-1}+q_{i+1}^{f-1}\right)+ \\
& \frac{2 a \tau_{T} \Delta t-h^{2} \tau_{q}}{h^{2}\left(\Delta t+\tau_{q}\right)} q_{i}^{f-2}-\frac{a \tau_{T} \Delta t}{h^{2}\left(\Delta t+\tau_{q}\right)}\left(q_{i-1}^{f-2}+q_{i+1}^{f-2}\right)- \\
& \frac{a(\Delta t)^{2}}{2 h\left(\Delta t+\tau_{q}\right)}\left(Q_{i+1}^{f-1}-Q_{i-1}^{f-1}\right)-\frac{a \tau_{T}(\Delta t)^{2}}{2 h\left(\Delta t+\tau_{q}\right)}\left(Z_{i+1}^{f-1}-Z_{i-1}^{f-1}\right)
\end{aligned}
$$

It should be pointed out that in the case of explicit scheme of the finite difference method the following stability condition should be fulfilled, namely

$$
\frac{h^{2}\left(\Delta t+2 \tau_{q}\right)-2 a \Delta t\left(\Delta t+\tau_{T}\right)}{h^{2}\left(\Delta t+\tau_{q}\right)} \geq 0
$$

Additionally, from the boundary conditions (12) and the initial conditions (13) it follows that $q_{i}^{0}=q_{i}^{1}=0$, where $i=0,1, \ldots, n$.

If the knowledge of the temperature is required, it is determined from equation (9). Thus, using appropriate differential quotients the following approximation of this equation can be obtained

$$
\frac{T_{i}^{f}-T_{i}^{f-1}}{\Delta t}=-\frac{1}{c} \frac{q_{i+1}^{f}-q_{i-1}^{f}}{2 h}+\frac{1}{c} Q_{i}^{f-1}
$$

and then for the internal nodes $i=1,2, \ldots, n-1$ one has

$$
T_{i}^{f}=T_{i}^{f-1}+\frac{\Delta t}{2 c h}\left(q_{i-1}^{f}-q_{i+1}^{f}\right)+\frac{\Delta t}{c} Q_{i}^{f-1}
$$


In the case of boundary nodes the dependence (5) should be taken into account, namely (cf. boundary conditions (12))

$$
-\lambda \frac{\partial T(x, t)}{\partial x}-\lambda \tau_{T} \frac{\partial^{2} T(x, t)}{\partial t \partial x}=0
$$

therefore

$$
-\lambda\left(\frac{\partial T}{\partial x}\right)^{f}-\lambda \frac{\tau_{T}}{\Delta t}\left[\left(\frac{\partial T}{\partial x}\right)^{f}-\left(\frac{\partial T}{\partial x}\right)^{f-1}\right]=0
$$

or

$$
\left(\frac{\partial T}{\partial x}\right)^{f}=\frac{\tau_{T}}{\Delta t+\tau_{T}}\left(\frac{\partial T}{\partial x}\right)^{f-1}
$$

For boundary node 0 one has

$$
\frac{T_{1}^{f}-T_{0}^{f}}{h}=\frac{\tau_{T}}{\Delta t+\tau_{T}} \frac{T_{1}^{f-1}-T_{0}^{f-1}}{h}
$$

and then

$$
T_{0}^{f}=T_{1}^{f}-\frac{\tau_{T}}{\Delta t+\tau_{T}}\left(T_{1}^{f-1}-T_{0}^{f-1}\right)
$$

while for boundary node $n$

$$
\frac{T_{n}^{f}-T_{n-1}^{f}}{h}=\frac{\tau_{T}}{\Delta t+\tau_{T}} \frac{T_{n}^{f-1}-T_{n-1}^{f-1}}{h}
$$

and then

$$
T_{n}^{f}=T_{n-1}^{f}+\frac{\tau_{T}}{\Delta t+\tau_{T}}\left(T_{n}^{f-1}-T_{n-1}^{f-1}\right)
$$

Taking into account the initial conditions (13) one has $T_{i}^{0}=T_{i}^{1}=T_{p}, i=0,1, \ldots$, $n$, of course.

Summing up, the knowledge of the local heat fluxes allows one to determine the temperature field at the set of points selected from the domain considered (in the case discussed at the nodes of differential mesh). 


\section{Results of computations}

The thin gold film of thickness $L=100 \mathrm{~nm}$ subjected to a short-pulse laser irradiation $\left(R=0.93, I_{0}=13.4 \mathrm{~J} / \mathrm{m}^{2}, t_{p}=0.1 \mathrm{ps}, \delta=15.3 \mathrm{~nm}\right.$ - cf. equation (11)) [1] is considered. Thermophysical parameters are the following $[1,5]$ : thermal conductivity $\lambda=315 \mathrm{~W} /(\mathrm{mK})$, volumetric specific heat $c=2.5 \mathrm{MJ} /\left(\mathrm{m}^{3} \mathrm{~K}\right)$, relaxation time $\tau_{q}=8.5 \mathrm{ps}$, thermalization time $\tau_{T}=90 \mathrm{ps}$. Initial temperature is equal to $T_{p}=300 \mathrm{~K}$.

The problem has been solved using the finite difference method under the assumption that $\Delta t=0.0001 \mathrm{ps}$ and $h=1 \mathrm{~nm}$.

In Figure 1 the heat flux distribution for times $0.2,0.3,0.4,0.5$ and $0.6 \mathrm{ps}$ is shown, while in Figure 2 the temperature distribution for the same moments of time is presented.

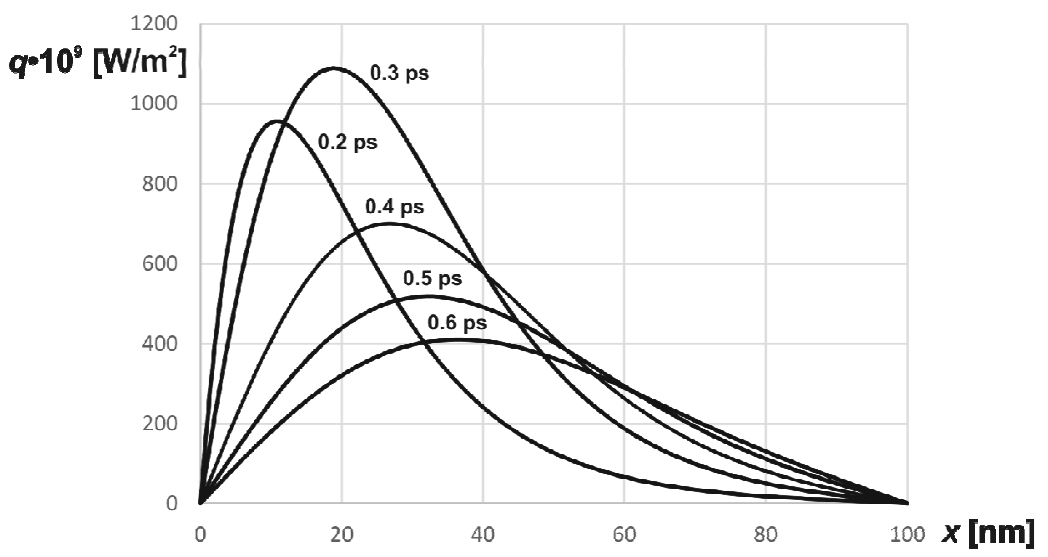

Fig. 1. Heat flux distribution

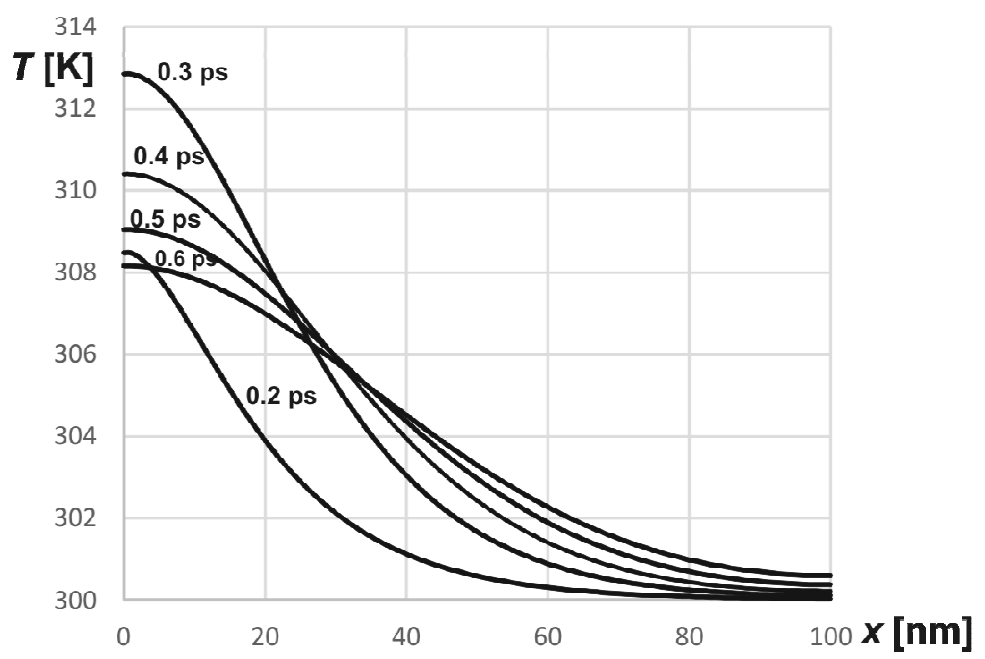

Fig. 2. Temperature distribution 


\section{Conclusions}

The dual-phase lag equation in which the heat flux is unknown is used to analyze the heat conduction in thin metal film subjected to the laser pulse. The problem is solved using an explicit scheme of the finite difference method for which the stability criterion is also derived. It should be pointed out that the results of computations have been compared with the results presented in [5] and they are practically the same.

\section{Acknowledgement}

This work is supported by the project No. 2012/05/B/ST8/01477 sponsored by the Polish National Science Centre.

\section{References}

[1] Chen J.K., Beraun J.E., Numerical study of ultrashort laser pulse interactions with metal films, Numerical Heat Transfer, Part A, 2001, 40, 1-20.

[2] Mochnacki B., Paruch M., Cattaneo-Vernotte equation. Identification of relaxation time using evolutionary algorithms, Journal of Applied Mathematics and Computational Mechanics 2013, 12(4), 97-102.

[3] Mochnacki B., Paruch M., Application of evolutionary algorithms for identification of dual phase lag model parameters, Scientific Research of the Institute of the Mathematics and Computer Science 2011, 10(1), 189-198.

[4] Majchrzak E., Mochnacki B., Suchy J.S., Finite difference model of short-pulse laser interactions with thin metal film, Computer Methods in Materials Science 2009, 9, 2, 316-322.

[5] Majchrzak E., Mochnacki B., Greer A.L., Suchy J.S., Numerical modeling of short pulse laser interactions with multi-layered thin metal films, CMES: Computer Modeling in Engineering and Sciences 2009, 41, 2, 131-146.

[6] Tzou D.Y., Macro- to Microscale Heat Transfer, The Lagging Behaviour, Taylor and Francis, 1997.

[7] Majchrzak E., Dziatkiewicz J., Kałuża G., Application of sensitivity analysis in microscale heat transfer, Computer Assisted Methods in Engineering and Science 2013, 20, 113-121. 